FORMATION Formation emploi

Revue française de sciences sociales

126 | Avril-Juin 2014

Qu'apprend-on des expérimentations sociales?

\title{
Le revenu d'autonomie pour les diplômés du supérieur : un premier pas vers une réforme du soutien financier de l'Etat à la jeunesse?
}

"Independent income for young graduates" : a first step towards a reform of financial support by the State to the youth?

Beihilfe (Revenu d'autonomie) für Hochschulabsolventen : Ein erster Schritt zur Reform der staatlichen Beihilfen für junge Menschen?

El ingreso de autonomía (RCA) para los graduados de la educación superior : ¿un primer paso hacia una reforma del apoyo financiero del Estado a la juventud?

Isabelle Borras et Nathalie Bosse

\section{OpenEdition}

\section{Journals}

Édition électronique

URL : http://journals.openedition.org/formationemploi/4211

DOI : 10.4000/formationemploi.4211

ISSN : 2107-0946

Éditeur

La Documentation française

Édition imprimée

Date de publication : 10 juillet 2014

Pagination : 119-136

ISSN : 0759-6340

Référence électronique

Isabelle Borras et Nathalie Bosse, «Le revenu d'autonomie pour les diplômés du supérieur : un premier pas vers une réforme du soutien financier de l'Etat à la jeunesse? », Formation emploi [En ligne], 126। Avril-Juin 2014, mis en ligne le 18 août 2014, consulté le 30 octobre 2020. URL : http:// journals.openedition.org/formationemploi/4211 ; DOI : https://doi.org/10.4000/formationemploi.4211 


\section{Le revenu d'autonomie pour les diplômés du supérieur : un premier pas vers une réforme du soutien financier de l'Etat à la jeunesse?}

ISABELLE BorRAS Ingénieur de recherches, économiste, directrice du Centre associé Céreq de Grenoble, CREG (Centre de Recherche en Economie de Grenoble), université Grenoble Alpes.

Nathalie Bosse Chargée d'études (spécialiste en sciences de l'éducation) au Centre associé Céreq de Grenoble, CREG (Centre de Recherche en Economie de Grenoble), université Grenoble Alpes.

Résumé

Le revenu d'autonomie pour les diplômés du supérieur : un premier pas vers une réforme du soutien financier de l'Etat à la jeunesse?

Lexpérimentation du Revenu Contractualisé d'Autonomie pour les Jeunes Diplômés est un des rares exemples illustratifs d'une tentative de réforme du système de soutien aux ressources des jeunes de moins de vingt cinq ans en France. Les résultats de l'évaluation qualitative de ce dispositif permettent de s'interroger sur la manière dont cette aide financière de l'Etat répond aux besoins de ses bénéficiaires, des jeunes diplômés du supérieur en difficulté, ainsi que sur ses limites.

Mots clés : expérimentation sociale, jeune, enseignement supérieur, revenu minimum, évaluation

Abstract

"Independent income for young graduates": a first step towards a reform of financial support by the State to the youth?

The experiment of the independent income for young graduates illustrates an attempt to reform the financial support to young people under 25 in France. We conducted a qualitative assessment of this experiment to analyse how the financial support by the State meets the needs of young people looking for their first job. We also point out the limits of the experiment. 
Keywords : social experiment, young person, higher education, minimum income guarantee, Evaluation

Journal of Economic Literature: H 53, I 38

Traduction : Auteures

En 2009, le Livre Vert ${ }^{1}$ du Haut-Commissariat à la Jeunesse proposait de «donner à tous les jeunes les moyens de prendre en main leur existence" (HCJ, 2009, p. 62). Ainsi, l'expérimentation du Revenu Contractualisé d'Autonomie pour les Jeunes Diplômés (RCA-JD) est l'un des rares dispositifs, en France, qui remet en cause le modèle « familialiste » de soutien aux ressources des jeunes avant $25 \mathrm{ans}^{2}$. Il consiste en effet à verser une allocation mensuelle garantie sur une année à des jeunes diplômés ayant au moins une licence, pendant leur recherche d'emploi, et à les accompagner dans cette recherche ( $c f$. encadré $\mathbf{1})$.

Bien que confidentielle, avec 500 places initialement prévues, la mise en œuvre du RCA-JD en 2011 et 2012 mérite donc une attention particulière. Elle constitue un terrain privilégié d'observation du passage des politiques aux pratiques de soutien à l'autonomie. Ce passage se heurte à un écueil principal (Van De Velde, 2012, pp. 61-62). L'autonomie ne s'impose pas : "Une politique d'autonomie ne peut se décréter, sans sincarner dans des mesures concrètes susceptibles d'atteindre sa cible. Son efficacité dépend in fine de sa capacité à inventer des outils d'indépendance en adéquation avec les besoins sociaux - qu'ils soient matériels, financiers, symboliques, institutionnels - au service d'un gain d'autonomie individuelle. "

Avec le RCA-JD, le législateur a-t-il inventé un outil d'indépendance en adéquation avec les besoins sociaux des jeunes à qui il était destiné, soit des jeunes diplômés du supérieur en difficulté sur le marché du travail ?

Afin de répondre à cette question, nous rappellerons tout d'abord le contexte d'une réforme des politiques de soutien aux ressources des jeunes, en France. Ce contexte éclaire les modalités de mise en œuvre du RCA-JD, les critères d'éligibilité retenus et l'inscription de l'allocation dans une logique de droits et de devoirs.

A partir des matériaux de l'évaluation qualitative du RCA-JD ( $c f$. encadré 2), nous analyserons ensuite les spécificités du public des bénéficiaires qui résultent de ces critères d'éligibilité. Au regard de ces spécificités, nous étudierons enfin la perception que les bénéficiaires ont du dispositif, à la fois de l'allocation et de l'accompagnement renforcé.

1. Issu de la commission de concertation lancée le 9 mars 2009, par Martin Hirsch, Haut-Commissaire à la Jeunesse, le Livre vert présente 57 propositions pour la jeunesse.

2. Deux autres dispositifs issus du Livre vert remettent également en cause ce modèle, le Revenu de Solidarité Active pour les jeunes actifs (RSA Jeunes) et l'expérimentation du Revenu Contractualisé d'Autonomie (RCA) auprès de jeunes non diplômés, dont le RCA-JD est une déclinaison pour les diplômés du supérieur. 


\section{Encadré 1 : L'expérimentation du Revenu Contractualisé d'Autonomie pour les Jeunes Diplômés}

La loi du 30/12/2009 et le décret d'application du 31/01/2011 fixent les conditions de mise en œuvre de l'expérimentation du RCA-JD. Celle-ci s'adresse à 500 jeunes volontaires sur six sites universitaires urbains : La Défense, Lille, Lyon, Marseille, Montreuil, Toulouse. Pour être éligibles, les jeunes doivent réunir quatre critères : être titulaires d'un diplôme de niveau bac +3 minimum, être inscrits depuis au moins six mois à Pôle emploi, ne pas être indemnisés, avoir moins de 24 ans à la signature du contrat.

Le RCA-JD combine une allocation garantie sur une année avec un accompagnement intensif à la recherche d'emploi confié à l'Apec (Association pour l'emploi des cadres).

Le montant de l'allocation est dégressif selon les revenus d'activités : il varie de 250 euros par mois (aucun revenu d'activité) à 10 euros (salaire au SMIC - salaire minimum interprofessionnel de croissance - à temps complet).

L'accompagnement par l'Apec comporte trois phases : « élaborer le projet premier emploi » (deux mois environ ; six entretiens en face-à-face, ateliers collectifs éventuels), « accéder au premier emploi » (jusqu'à dix mois ; entretiens individuels tous les quinze jours, sessions collectives éventuelles), « suivre dans l'emploi » (trois mois, avec deux ou trois entretiens)

\section{Un contexte peu propice aux réformes}

L'autonomie désigne la capacité des jeunes à mener, par eux-mêmes, leur choix de vie et à devenir adultes, mais aussi à s'assumer seuls sur le plan financier et matériel. Durant ces dernières décennies, la jeunesse et son autonomie sont régulièrement mises à l'agenda des politiques publiques en France, en lien avec l'évolution des conditions de vie des jeunes adultes. L'allongement et la massification des études, conjugués aux difficultés d'insertion professionnelle, ont reculé l'âge d'entrée dans la vie active et l'accès à des revenus du travail stables et sources d'autonomie matérielle (Galland, 2011 ; Nicole-Drancourt, RoulleauBerger, 2006). Ainsi, des jeunes de plus en plus " âgés ", et donc au désir d'autonomie croissant, restent désormais dépendants des soutiens familiaux. Ils n'ont en effet pas accès au système de protection sociale qui, en France, est réservé aux plus de 25 ans. Ce contexte est également propice au développement de la pauvreté juvénile, phénomène plus nouveau et préoccupant pour l'action publique ${ }^{3}$.

3. En témoigne cet extrait du rapport "Priorité Jeunesse ", du 21 février 2013, du Comité Interministériel de la Jeunesse : "Les difficultés d'accès à l'emploi des jeunes se répercutent sur leurs conditions de vie. Le risque de pauvreté est d'autant plus préoccupant que la protection sociale dont bénéficient les jeunes est inférieure à celle des adultes sur certains aspects : assurance-chômage et revenus minima garantis notamment (...) En 2000, le taux de pauvreté des jeunes de 18 à 24 ans sélevait à 19,9\%; il atteint 22,5\% en 2009 alors quill sélève à 13,5\% pour l'ensemble de la population.» 
Dans une société qui valorise de plus en plus la liberté individuelle, l'enjeu d'une autonomie accrue de la jeunesse fait consensus. Les débats portent sur les réponses politiques à apporter. Faut-il, et comment, réformer les systèmes de soutien aux ressources de la jeunesse en France ? L’enjeu est considérable : il s'agit de "dé-familialiser » le système. De nombreux textes, rapports gouvernementaux, débats parlementaires ${ }^{4} \ldots$ formulent des propositions allant dans ce sens : mise en place d'allocations ou de dotations, universelles ou sous conditions de ressources, versées aux jeunes sur critères d'âge ou aux étudiants, et plus ou moins fléchées sur des usages précis, comme la formation, ou libres d'utilisation... Les rapports les plus récents évoquent les expériences à l'étranger. Celui du HCJ (2009) étudie le système de soutien aux étudiants au Danemark où l'Etat se porte garant de l'autonomie dès 18 ans via une allocation qui permet aux jeunes d'expérimenter des allers et retours entre études et emploi et de "maitriser leurs choix biographiques". Cette indépendance par l'Etat, caractéristique des systèmes socio-démocrates de protection sociale, s'oppose à une indépendance par autrui via le groupe familial, caractéristique des systèmes conservateurs corporatistes, typiquement français, mais aussi à l'indépendance par la dette et l'emploi, d'inspiration libérale et anglo-saxonne (Van De Velde, 2008).

Jusqu'en 2009, toutes les propositions restent lettre morte. Les politiques de soutien à l'autonomie se résument à "quinze ans de préconisations inabouties" (Cicchelli, 2012, p. 65), et à "une mise à l'agenda affichée et à une non-décision silencieuse " (Chevalier, 2012, p. 188). Les raisons de l'échec résideraient dans l'incohérence des changements proposés avec le modèle "familialiste " français, et dans le succès de deux arguments consensuels dans la société française en défaveur d'une réforme : la crainte du délitement du lien familial et la peur de l'assistanat de la jeunesse (Chevalier, op.cit. pp. 137138). Ce n'est que depuis 2009, avec la concrétisation de deux propositions du Livre Vert, qu'apparait une ébauche de réforme, selon une stratégie en deux temps : un système cible de "soutien aux revenus des jeunes universel, unifié et défini à partir des besoins des jeunes", associé à "des mesures immédiates et au lancement d'expérimentations". La proposition 37 qui vise à «soutenir les ressources des jeunes pour améliorer leur accès à une formation ou à un emploi » débouche ainsi sur l'expérimentation du RCA-JD adressée à 500 jeunes de 18 à 23 ans, possédant au moins une licence, inscrits depuis plus de six mois à Pôle emploi et non indemnisés5.

4. Il s'agit de rapports commandés par le gouvernement (Charvet, CGP, 2001 ; De Foucault et Roth, CGP, 2002 ; Boissard, CAS, 2007 ; Livre Vert, 2009) mais aussi de textes partisans issus de partis politiques, syndicats, Think-tank... (Bocquet, 2000 ; Brin, 2001 ; UNEF, 2009 ; TERRA NOVA, 2010). La liste est non exhaustive. Pour une présentation et une analyse systématique, voir Chevalier (2012) et Cicchelli (2012).

5. Elle débouche aussi sur l'expérimentation du RCA pour 5000 jeunes non diplômés âgés de 18 à 22 ans. La proposition 38, qui vise à "soutenir les revenus des jeunes en emploi sans discrimination par rapport aux plus de 25 ans", conduit à une mesure immédiate, le RSA jeunes. 


\section{Encadré 2 : L'évaluation du Revenu contractualisé d'autonomie pour les jeunes diplômés : méthodologie}

L'évaluation a été soutenue par le Fonds d'expérimentations pour la jeunesse (FEJ) mis en œuvre par le ministère en charge de la Jeunesse. La méthodologie préconisée était qualitative, en lien avec le faible volume visé (500 places offertes). Elle mobilise trois sources :

- Données Apec (Association pour l'emploi des cadres) : des traitements statistiques des fichiers de l'Apec ont été réalisés afin de caractériser les profils des 276 bénéficiaires (sexe, âge, formation initiale) et leurs parcours (durée et issue de l'accompagnement, montants des allocations versées...)

- Entretiens Apec et Pôle Emploi : 28 entretiens semi-directifs en face-à-face ont été menés auprès des professionnels impliqués dans le dispositif sur les six sites expérimentaux. Une première série d'entretiens a porté sur la mise en œuvre du RCA-JD et le ciblage des jeunes : fonctionnement du partenariat entre Pôle emploi et l'Apec, critères et modalités de repérage et de prise de contact avec les jeunes, perception des attentes des jeunes, motifs de refus repérés par les consultants... Une seconde série d'interviews a porté plus spécifiquement sur l'accompagnement et l'allocation : contenu, déroulement et finalités de l'accompagnement, la place de l'allocation dans le dispositif et son utilité du point de vue des consultants, profils des jeunes suivis...

- Réalisation d'une enquête par questionnaire et d'une enquête par entretiens semidirectifs auprès des allocataires entrés dans le dispositif entre mars et juillet 2011. Les deux enquêtes ont été menées en juin et juillet 2012. A cette date, seuls quelques jeunes étaient encore accompagnés. Elles ont abordé des thèmes communs : le parcours de formation et la situation personnelle à la fin des études, la recherche d'emploi avant le RCA-JD, les motifs d'entrée dans le dispositif, le déroulement de l'accompagnement, l'usage de l'allocation, les perceptions de l'impact du dispositif sur les parcours, la situation à la fin de l'accompagnement et à la date de l'enquête, l'opinion globale sur le parcours, ainsi que des renseignements individuels (sexe, CSP - catégorie socio-professionnelle - du père...). Les entretiens (d'une durée comprise entre 40 minutes et une heure) ont été retranscrits et ont fait l'objet d'une analyse thématique. Les réponses issues du questionnaire ont donné lieu à un traitement statistique descriptif :

L'enquête par questionnaire : Les 165 jeunes répondants à cette enquête administrée par internet (taux de réponse $60 \%$ ) sont représentatifs des 276 bénéficiaires sur des critères de genre ( $67 \%$ et $65 \%$ respectivement de jeunes femmes), de sites universitaires, d'issue du RCA (part des jeunes en emploi...). La première population est un peu plus diplômée : sur-représentation des « bac +5 » (53\% pour $45 \%$ dans la population des allocataires) et des jeunes accompagnés 12 mois ( $27 \%$ pour $23 \%$ ).

L'enquête par entretiens semi-directifs a été conduite par téléphone auprès de 40 jeunes : 24 femmes et 16 hommes, 15 jeunes accompagnés un an, 15 entre 6 et 12 mois, 7 entre 3 et 6 mois, et 3 restés dans le dispositif moins de 3 mois. La moitié des 40 interviewés possèdent un diplôme de niveau bac $+5,16$ un diplôme de niveau bac +3 et 4 déclarent un niveau d'études équivalent à $\mathrm{bac}+4$. 
Avec le RCA-JD, la réforme avance donc à petits pas. Il est expérimenté sur des territoires délimités. Les 500 places concernent six territoires universitaires urbains (75 à Créteil, La Défense et à Lille, 100 à Lyon et Marseille et 50 à Toulouse). Il est soumis à une évaluation avant une éventuelle généralisation, selon le principe même des expérimentations sociales issues du Livre vert. Avec le RCA-JD, la réforme ne remet pas en cause les règles existantes de soutien aux ressources des jeunes. L'aide de l'Etat ne concerne ici que les « oubliés " de l'action publique, les moins de 25 ans ni en emploi ni en formation. En sont donc exclus les étudiants qui dépendent des transferts familiaux et des bourses, mais aussi les jeunes diplômés de plus de 25 ans en recherche d'emploi éligibles aux minima sociaux ou ceux indemnisés par l'assurance chômage ou touchant une indemnité de service civique. Le changement graduel est adapté à "un contexte dans lequel il est possible d'introduire de nouvelles règles formelles" (le versement d'une allocation avant 25 ans) "mais difficile de réformer celles existantes" (le système "familialiste" de soutien aux étudiants) ${ }^{6}$.

Enfin, avec le RCA-JD, la réforme s'inscrit dans le "paradigme commun" des réformes des politiques de l'emploi dans les pays de l'OCDE (Organisation de coopération et de développement économiques), qui est celui de l'activation de la protection sociale (Barbier, 2006). Pour l'OCDE, "les stratégies d'activation consistent principalement à faire appliquer les principes de disponibilité à l'emploi et d'obligations mutuelles, qui impose aux allocataires de rechercher activement du travail et d'améliorer leur employabilité, en contrepartie d'un service efficace de réinsertion et de paiement de prestations " ${ }^{7}$. Ainsi le RCA-JD a pour finalité l'insertion professionnelle: "un système qui ne répondrait pas aux besoins des jeunes abordés transversalement manquerait ses objectifs. Il les manquerait aussi, sìl est abordé indépendamment des mesures nécessaires pour améliorer l'insertion professionnelle des jeunes " (HCJ, op.cit, p. 62). Il s'adresse à des jeunes en difficulté pour qui le versement de l'allocation est conditionné à l'engagement contractuel de suivre un accompagnement renforcé à la recherche d'emploi, confié à l'Agence pour l'emploi des cadres (Apec). Le montant mensuel maximum de l'allocation est de 250 euros, mais un montant réduit peut être versé en fonction des revenus d'activités, pour inciter les jeunes au travail. Ces choix, qui tiennent à distance le spectre de l'assistanat de la jeunesse, légitiment l'expérimentation.

\section{Un public de bénéficiaires très spécifique}

Le contexte précédent éclaire les critères d'éligibilité retenus pour la mise en œuvre du RCA-JD. Afin de toucher des diplômés du supérieur rencontrant des difficultés à trouver

6. Cf. Chevalier (op. cit.). Voir aussi Palier, Surel (2010) sur les approches du changement dans l'analyse des politiques publiques.

7.http://www.oecd.org/fr/els/emp/politiquesactivesdu marchedutravailetstrategiesdactivation.htm 
leur premier emploi, les jeunes éligibles devaient réunir deux premiers critères : être titulaires d'un diplôme de niveau bac +3 au minimum et être inscrits depuis au moins six mois à Pôle Emploi. Par ailleurs, afin d'écarter des jeunes pouvant devenir éligibles au RSA, ils devaient avoir moins de 24 ans à la signature du contrat. Enfin, le RCA-JD n'a pas été proposé aux jeunes percevant une indemnité de chômage ou de service civique. Conséquence de ces critères restrictifs, l'objectif en nombre d'entrées dans le dispositif n'a pu être atteint. On est également en présence d'une population particulière : des jeunes très diplômés, soutenus financièrement par leurs parents. La moitié déclare néanmoins occuper un emploi alimentaire, le plus souvent à temps partiel, en attendant de trouver celui correspondant à leur projet professionnel. Les entretiens révèlent que leurs difficultés dans la recherche de cet emploi sont réelles. En effet, ils apparaissent non préparés à la recherche d'emploi et expliquent s'être retrouvés isolés durant cette période, sans interlocuteur pouvant leur apporter conseil.

\subsection{Des critères d'éligibilité restrictifs}

Sur chacun des six territoires de l'expérimentation, Pôle emploi et l'Apec se sont organisés pour identifier les jeunes éligibles, les informer sur leur droit d'accès à ce nouveau dispositif et, parfois, tenter de les convaincre. Pôle Emploi, prescripteur du RCA-JD, a procédé à des extractions informatisées des listes de jeunes réunissant l'ensemble des critères. Les jeunes ont ensuite été invités ou parfois convoqués à des réunions d'information collective.

Au final, 276 contrats ont été signés au lieu des 500 prévus. Pour les professionnels de Pôle Emploi et de l'Apec interviewés ( $c f$. encadré 2), la difficulté à atteindre l'objectif de 500 entrées s'explique surtout par le nombre réduit de jeunes réunissant les quatre critères. Deux de ces critères ont particulièrement suscité des réserves. Le premier est l'ancienneté de six mois d'inscription comme demandeur d'emploi. Le dispositif passe alors à côté des nombreux jeunes qui n'ont pas entrepris cette démarche. Selon une enquête commandée par le Haut-Commissaire à la Jeunesse en $2009^{8}$, seulement un jeune sur deux en recherche d'emploi est inscrit à Pôle Emploi quelques mois après la fin des études. Le second critère est l'âge limite de 24 ans : il est souvent mentionné que l'association des critères d'âge, de durée d'inscription et de diplôme débouche essentiellement sur des jeunes au "parcours sans faute " (un consultant Apec) ${ }^{9}$.

Le vivier de jeunes éligibles était manifestement très limité, ce que confirme une estimation d'une population comparable à celle éligible au RCA-JD à partir de l'enquête Génération 2007 du Céreq. Cette enquête recense 175000 jeunes sortants du système

8. Enquête sur l'insertion des jeunes diplômés, menée par la Sofres, auprès d'un échantillon représentatif d'étudiants diplômés issus de BTS, DUT (respectivement brevet de technicien supérieur et diplôme universitaire de technologie), licence professionnelle et master. 1006 jeunes ont été interrogés quelques mois après leur sortie.

9. En italique dans le texte, les propos des personnes interviewées. 
éducatif en 2007, possédant au moins une licence (docteurs exclus). Parmi eux, 15000 $(8,9 \%)$ ont recherché plus de six mois un emploi avant leur premier emploi et 6250 $(3,6 \%)$ avaient moins de 24 ans après six mois de recherche. Ces 6250 jeunes constituent une borne haute de la population éligible au niveau national dans l'enquête Génération 2007, car elle englobe des jeunes non inscrits à Pôle emploi.

Cette difficulté à atteindre l'objectif rappelle le RSA jeunes ( $c f$. note 5) pour lequel le choix d'un critère d'accès trop restrictif - " avoir travaillé deux ans dans les trois dernières années" - explique "qu'à la fin de 2010, le bilan est d'environ 8000 jeunes pris en charge pour un objectif initial de 160000 "(Vulbeau, 2012). L'incapacité des pouvoirs publics à définir une population cible interroge. Visait-on principalement un effet d'annonce ? $\mathrm{Ou}$ bien cette prudence dans la mise en œuvre résulte-t-elle d'une méconnaissance des publics et de leurs besoins?

\subsection{Un public très diplômé et issu de milieux plutôt favorisés}

Les 276 jeunes entrés dans le dispositif sont majoritairement des femmes (65\%) et sont proches de l'âge limite du RCA-JD : les trois quarts ont en effet 23 ans à la signature du contrat. $55 \%$ détiennent un diplôme de niveau bac $+3 / 4$ et $45 \%$ de niveau bac +5 . Plus de la moitié sortent de l'université (53\%). Les autres sont diplômés d'écoles de spécialité $(16 \%)$, d'ingénieur (14\%), de commerce et de gestion (10\%). Ils sont issus de formations variées, même si certaines sont surreprésentées, notamment arts et création (11\% des bénéficiaires), environnement et écologie (8\%), ou encore communication et journalisme $(7 \%)^{10}$.

Dans l'enquête par questionnaire à laquelle 165 jeunes ont répondu ( $c f$. encadré 2), plus de la moitié (56\%) déclarent vivre chez leurs parents pendant la recherche d'emploi, $26 \%$ seuls et $18 \%$ en couple. La plupart sont soutenus par les parents durant cette période de transition : $90 \%$ affirment pouvoir compter sur leurs proches pour subvenir à leurs besoins et $72 \%$ perçoivent des ressources de leur famille. Ils sont par ailleurs souvent issus de milieux sociaux plutôt favorisés (39 \% ont un père cadre), ce qui est caractéristique des jeunes ayant poursuivi des études supérieures ${ }^{11}$. Néanmoins, quelques-uns montrent des signes de précarité sociale lorsqu' ils déclarent "ne pas toujours avoir mangé à leur faim » (13 jeunes, $8 \%$ des répondants), "renoncé à des achats de première nécessité » ou "à des soins " (23 à $25 \%$ des répondants).

Autre spécificité, ils sont nombreux à rechercher leur premier emploi tout en travaillant, mais les revenus perçus sont trop faibles pour ouvrir des droits à l'assurance chômage. Une

10. Les autres sont diplômés en marketing (6\%), chimie (5\%), sciences et vie de la terre, biologie (4\%), tourisme-hôtellerie ( $4 \%$ ), alimentaire (4\%), langues (4\%), architecture design industriel (3\%), électronique, électrotechnique, automatisme (3\%)... (Source : Apec).

11. Selon le Céreq (2012), $19 \%$ des sortants du système éducatif en 2007 ont un père cadre. Ce pourcentage passe à $28 \%$ pour les sortants de licence et $44 \%$ pour les sortants de master 2 . 
moitié des 165 répondants à l'enquête déclare avoir travaillé pendant leur recherche d'emploi, de manière régulière ou ponctuelle. Les entretiens révèlent que les postes occupés, très souvent à temps partiel, parfois saisonniers, sont divers : manœuvre dans le BTP (bâtiment et travaux publics), téléprospecteur, animatrice en centres de loisirs, chargée d'accueil dans une banque, serveuse, soutien scolaire... Pour les jeunes, occuper ces " jobs " ${ }^{12}$ permet d'améliorer leur quotidien, de soulager leurs parents en gagnant un peu d'argent, de se sentir moins dépendants de ces derniers, ou encore de s'occuper : "J'ai fait des petits jobs par-ci, par-là parce que jen avais besoin, c'était des petits jobs alimentaires (...) J'habitais chez ma mère, elle ne mia pas donné d'argent. Je ne payais pas de loyer mais j'avais un job à côté. " (Lise, bac + 5, langues), "C'était pour gagner un petit peu d'argent. J'ai travaillé un mois et demi comme intérimaire, j’étais chargé d'accueil à la Caisse d'Epargne (...) Je n’en avais pas autant besoin que quelqu'un qui n'est pas aidé par ses parents comme je l'ai été. En étant chez mes parents... ils ne mont pas fait payer le loyer ou... J'ai participé un petit peu aux courses, mais c'était aussi pour faire quelque chose et rester dans une dynamique, pas forcément que faire de la recherche d'emploi, rester tout le temps à la maison ou me déplacer pour quelques entretiens " (Jérémy, bac +5 , environnement).

La capacité des jeunes diplômés à occuper ces " jobs ", dans la continuité des jobs étudiants (Dmitrijeva et alii, 2013) révèle le véritable enjeu de la recherche d'emploi : l'accès à un emploi de qualité, loin d'être garanti malgré le diplôme ; en effet, trois ans après la fin des études, " un tiers des emplois (des diplômés du supérieur) se situe dans la classe des bons emplois, $41 \%$ sont des emplois de qualité moyenne et $25 \%$ de qualité médiocre (...) les jeunes ayant eu un accès direct à l'emploi ont plus de chances d'occuper un emploi de bonne qualité trois ans après (...) les trajectoires où l'accès à l'emploi est différé ou difficile conduisent à un emploi de moins bonne qualité " (Moncel, 2012).

\subsection{Une recherche d'emploi difficile}

La plupart des bénéficiaires ont obtenu leur dernier diplôme entre juin et septembre 2010, et sont entrés dans le dispositif au cours du premier semestre 2011. Les jeunes interviewés décrivent ainsi plusieurs mois de recherche infructueuse après la fin de leurs études. Ils déclarent trouver peu d'offres d'emploi correspondant à leur projet professionnel.

Plusieurs explications sont avancées. Certains recherchent dans des métiers très pointus, offrant peu de débouchés : l'un vise, par exemple, un emploi de phytosociologue ${ }^{13}$, une autre souhaite devenir interprète en langues des signes. Plus généralement, les jeunes déclarent prospecter dans des secteurs qui recrutent peu, bien qu'ils présentent des profils variés : infographiste, ingénieur en environnement, conseiller en insertion professionnelle... L'absence de mobilité géographique de certains apparaît en outre comme un

12. On parle de « jobs » pour les différencier des emplois recherchés par les jeunes.

13. La phytosociologie est la discipline botanique qui étudie les communautés végétales. 
facteur aggravant. Autre obstacle, les jeunes ont l'impression de ne pas correspondre aux profils recherchés par les entreprises et c'est le manque d'expérience qui est évoqué comme la principale difficulté : "Après mon bac scientifique, j’ai suivi des études de psychologie donc la licence et ensuite le master professionnel de psychologie du travail et des organisations (...) Je voulais plutôt être conseillère en insertion (...) [Les difficultés], je dirais le manque d'offres et je dirais le fait qu'ils demandaient au moins deux à trois ans d'expérience, ça coince " (Julia, bac +5 , psychologie); "Je cherchais un poste d'ingénieure d'études en biologie (...) Je n'ai jamais décroché d'entretien et c'est une grosse difficulté (...) Je n'avais pas l'expérience, ça on me l'a très très souvent reproché, je n'avais pas assez d'expérience dans le domaine " (Anna, bac + 5, biologie).

Parfois, une formation incomplète vient majorer les difficultés des jeunes : une première année de master seulement, un master recherche qui aurait dû déboucher sur une thèse de doctorat, ou encore une licence qui devait se poursuivre sur un master... La plupart du temps, ces cursus « inachevés » ne découlent pas d'un choix délibéré de la part des jeunes. Ces derniers n'ont, par exemple, pas été acceptés dans les formations souhaitées. Ils ne possèdent alors pas le niveau de qualification exigé par les employeurs, le niveau requis pour passer des concours, ou détiennent un diplôme qui ne prépare pas à une insertion directe sur le marché du travail : "J'ai obtenu mon master de microbiologie en 2010 (...) J'avais tenté d'avoir une thèse et en fait je n'ai pas eu le financement pour pouvoir la faire. Du coup, ça s'est arrêté là et j'ai essayé de trouver du travail. " (Anna, bac + 5, biologie)

L'absence de projet professionnel clairement défini est également mise en avant par quelques jeunes, qui se retrouvent ainsi perdus au moment de rechercher un emploi : "Je ne savais pas où chercher. C'était ça le gros dilemme, je n'avais pas de (...) j'avais envie de travailler mais je ne savais pas dans quoi (...) En fait, jétais vraiment perdue. " (Lise, bac +5 , langues)

Ces difficultés évoquées par les jeunes sont, de manière plus générale, à relier à leur absence de préparation à la recherche d'emploi. Ainsi, ils expliquent ne pas savoir quelles démarches entreprendre. Ils soulignent à la fois leur manque de méthodes, mais aussi la difficulté de gérer leur appréhension ou leur stress, par exemple au moment de contacter les entreprises ou lors d'un entretien d'embauche: "Je suis allée jusqu'au master en biologie "Expression génétique et protéines recombinantes" (...) J’espérais entrer dans une équipe de recherche publique en fait (...) C'était assez difficile, il n'y avait pas d'offres du tout. Et du coup, je ne savais pas comment chercher, javais du mal à contacter les gens, tout ça. Je n'avais pas trop de connaissances. " (Amélie, bac +5 , biologie)

Souvent, ils confient se sentir démunis et isolés, malgré les conseils et le soutien de l'entourage. Ils décrivent un accompagnement via Pôle emploi très limité avant d'entrer dans le RCA-JD, et pas toujours adapté à leur profil. Ils soulignent notamment une méconnaissance de leurs diplômes et des métiers visés : "Je n'étais pas cadré, il n’y avait rien pour m’orienter, cétait une démarche de recherche d'emploi en solitaire. " (Lucas, bac + 3, design) ; "Je n'étais pas du tout satisfaite de Pôle emploi (...) L'emploi que je recherchais, pour eux, il 
n'existait pas, n'était pas dans leur liste, leur fameuse liste d'emplois possibles. Donc, quand je disais que je cherchais un emploi de médiatrice culturelle, on menvoyait forcément dans le domaine très large de la culture. " (Romane, bac + 4, histoire) ; "J'ai eu un entretien, chez Pôle emploi, collectif, pour qu'on me présente mon référent on va dire. Et tous les mois, je devais avoir un rendez-vous, et jusqu'à mars je n'ai pas eu de rendez-vous individuel pour faire mon suivi de recherche d'emploi. Du coup, j'ai été tout de suite autonome on va dire" (Léa, bac + 3, agroalimentaire).

Le critère d'au moins six mois d'inscription à Pôle emploi a donc conduit vers le dispositif des jeunes dont les difficultés entrent en résonance avec plusieurs " profils types » identifiés par l'AFIJ (2012) : jeunes en situation d'échec par rapport à leur projet initial (en échec en master 1 et titulaires d'une licence généraliste...), en recherche de projet professionnel (les sortants de filières généralistes ou professionnelles à faibles débouchés, jeunes en réorientation...), ou encore rencontrant des difficultés particulières (exigences découlant du projet professionnel ou personnel - lieu de travail, salaire d'embauche, fonction - problèmes de comportement...), etc. Pour l'AFIJ, un jeune sur deux issu du supérieur serait concerné par ces difficultés.

\section{Un accompagnement à la recherche d'emploi, I'allocation en prime}

Les volontaires pour le RCA-JD sont invités à signer un contrat avec l'Apec, qui fixe leurs obligations de recherche d'emploi. Les résultats issus de l'évaluation permettent de comprendre les motifs qui ont incité les jeunes à entrer dans le dispositif et les besoins auxquels ce dernier répond. Ainsi, pour $83 \%$ des 165 répondants à l'enquête par questionnaire, l'allocation de 250 euros a joué un rôle important dans leur décision de s'engager, presque autant que la perspective d'être accompagné ( $89 \%)$. Cette aide financière ne permet pas aux jeunes de prendre leur indépendance économique, mais représente une rentrée d'argent qui ne se refuse pas : "Ce n'est pas rien 250 euros par mois. " (Adrian, bac +5 , écologie) Cependant, très en difficulté dans leur recherche d'emploi, les diplômés étaient surtout en attente du type d'accompagnement proposé dans le cadre du RCA-JD : « $C e$ qui mintéressait, c'était surtout de trouver du conseil, je m’intéressais surtout à la qualité du conseil. » (Emma, bac + 5, arts décoratifs).

\subsection{L'allocation facilite la recherche d'emploi et couvre une partie des dépenses quotidiennes}

Pour les bénéficiaires interviewés, l'allocation est bienvenue étant donné qu'ils ne percevaient, jusqu'alors, aucune forme d'aide financière. Elle vient en effet combler un vide dans le système de protection sociale de ces jeunes : "J'ai trouvé ça très bien parce qu’à l'époque, je n'avais pas encore 25 ans, donc je ne pouvais rien toucher, et c'est vrai quil y a 
beaucoup de gens dans mon cas aussi et on a droit à aucune aide. "(Amélie, bac +5 , biologie) Cependant, son importance est variable en fonction des situations, car aucun critère social ou de ressource n'a joué pour l'éligibilité. Ainsi, au moment de la signature du contrat, $29 \%$ des allocataires signalent " avoir des difficultés financières et vraiment besoin de l'allocation $»^{14}, 34 \%$ "pensent pouvoir se consacrer davantage à la recherche d'emploi avec cette allocation». Pour $36 \%$, elle "représente un plus sans être indispensable». $2 \%$ seulement "n'en ont pas du tout besoin " (enquête par questionnaire). Le montant de 250 euros est le plus souvent considéré comme correct par les jeunes pouvant compter sur leur famille: "Pour moi, c'était plus que correct, sachant que j'avais mes parents qui suivaient derrière. " (Chloé, bac +5 , droit) Mais d'autres précisent aussi que cela n'est pas suffisant pour couvrir tous leurs besoins: "Après, au niveau de la somme, c'est assez difficile à juger. C'est-à-dire que ça ne permet pas de vivre mais ça permet d'améliorer un peu le quotidien, de payer les courses, de payer les choses qui reviennent un peu tous les mois» (Marion, bac +5 , chimie).

Le montant de l'allocation versée dépend des durées d'accompagnement et d'éventuels revenus d'activités ( $c f$. encadré $\mathbf{1}$ ). Au total, $20 \%$ des 276 allocataires ont perçu moins de 500 euros et $23 \%$ plus de 2000 euros (Données Apec). Pour les jeunes interviewés, le versement de l'allocation a permis d'améliorer les conditions de recherche d'emploi, en contribuant directement aux dépenses qui lui sont liées : impression des CV, inscription payante sur des sites de recherche d'emploi, envoi de courriers, paiement des billets de train ou de l'essence pour se rendre à des entretiens... : "J'ai utilisé cet argent pour acheter des vêtements pour être présentable lors des entretiens. Après, ça m’a permis d'acheter des enveloppes, du papier pour mes candidatures. J'ai acheté des manuels pour progresser au niveau du dessin sur ordinateur. Franchement, cette aide a été bénéfique " (Maxime, bac + 3, électrotechnique), "Elle m'a été utile deux fois pour louer un véhicule pour me rendre à un entretien et plusieurs fois aussi pour prendre le train " (Charlotte, $\mathrm{bac}+3$, chimie).

Mais l'allocation a plus largement participé à l'ensemble des dépenses quotidiennes : "Un peu de tout. Je m'en suis servi à la fois pour la recherche d'emploi, pour les frais de timbres, puis éventuellement les déplacements. Du fait que j’ai passé des concours, j’étais obligé de me déplacer. Puis sinon, plutôt dans la vie quotidienne, et occasionnellement pour ce qui était plus des extras" (Clément, bac +5 , chimie). Certains, vivant seuls, l'ont utilisée en partie pour "payer le loyer " et les factures diverses. Par exemple, pour une jeune femme, sans aucun soutien familial, la somme perçue a été exclusivement destinée aux dépenses quotidiennes : "Ce n'était pas pour la recherche d'emploi, ça c'est sûr. C'était pour la vie quotidienne (...) Le remplissage de mon frigo " (Louise, bac +3 , langue des signes). D'autres utilisations de l'allocation sont évoquées, comme le financement du

14. La réponse dépend de la situation personnelle : ils sont $25 \%$ parmi les jeunes vivant chez les parents, $30 \%$ parmi ceux qui vivent en couple et $36 \%$ parmi ceux vivant seuls (enquête par questionnaire). 
permis de conduire, un déménagement... De manière plus marginale, l'allocation a financé sorties et loisirs, et certains déclarent l'avoir épargnée : "Je pense que j’en ai bien profité pour faire les magasins aussi " (Anaïs, bac +5 , communication), "Je n'en ai pas dépensé beaucoup en fait étant donné que je suis chez mes parents. Je les ai surtout placés" (Julien, bac + 4, géographie).

Les entretiens révèlent également que cette somme a permis à certains jeunes de se sentir moins dépendants matériellement et symboliquement de leurs parents : "Ça me permettait aussi, même si mes parents ne disaient rien, de ne pas être entièrement à leur charge, de me dire "tiens je peux payer les courses", même si ce n'est qu'une fois par mois mais voilà, je participe "

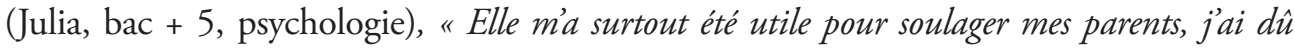
ramer deux ans (...) Ce n'est pas une situation très agréable d'être complètement dépendant de ses parents financièrement " (Noémie, bac +3 , design).

Néanmoins, les jeunes interviewés n'expriment pas l'attente légitime d'une vraie « allocation d'autonomie ", telle que revendiquée par l'UNEF (Union nationale des étudiants français, 2009). Ceci s'explique en partie par la composition des publics, proches de l'âge médian de la décohabitation des jeunes français et pour qui vivre chez ses parents est jugé normal. "L'accès à l'indépendance individuelle est non seulement une affaire de ressources, étatiques et salariées, mais également de normes culturelles" (Van de Velde, 2012). Des jeunes plus sensibles au sujet, ayant dé-cohabité et de milieux modestes, étaient a priori peu présents dans ce dispositif ; ils étaient en effet contraints d'occuper des " jobs " alimentaires prenants et donc non disponibles pour l'accompagnement. Une autre explication réside dans le faible montant de l'allocation, présentée aux bénéficiaires d'abord comme une aide à la recherche d'emploi et non comme une allocation d'autonomie.

\subsection{L'accompagnement rompt la solitude de la recherche d'emploi}

D'une durée de douze mois, l'accompagnement articulé à l'allocation est intensif, avec de fréquents entretiens entre le jeune et son consultant. Structuré en trois phases (cf. encadré 1), son objectif est de valider le projet professionnel du jeune et de définir la meilleure stratégie d'accès à l'emploi et le plan d'action le plus adapté. Le protocole d'accompagnement a été adapté par les consultants en fonction des situations individuelles, mais la fréquence des rendez-vous a été respectée : six entretiens d'environ une heure les deux premiers mois ; au-delà, les bénéficiaires déclarent un à quatre contacts par mois (enquête par questionnaire).

Pour les jeunes, cette proposition d'accompagnement arrive après plusieurs mois de chômage, à un moment où ils ressentent doutes et découragement. Elle apparait alors comme une possibilité de rompre la solitude de la recherche d'emploi : "Quand on est toujours tout seul à chercher, à un moment, c'est un peu désespérant on va dire. Donc je me disais quiavec des rencontres régulières avec quelquiun, c'était plus motivant" (Romane, bac +4 , histoire). Ainsi, le suivi régulier, la fréquence des entretiens avec le consultant, l'accompagnement 
individualisé,... apparaissent comme autant d'éléments ayant suscité leur intérêt : " Dans ma tête, c'était Pôle emploi mais en mieux, avec quelqu'un qui nous suivait plus régulièrement (...) Il sarrêtait vraiment sur le dossier de chacun et essayait de faire un suivi personnalisé " (Charlotte, bac +3 , chimie).

Les jeunes sont également entrés dans le dispositif dans l'attente de recevoir des conseils pratiques, d'acquérir des méthodes pour organiser et optimiser leur recherche d'emploi : "Je me disais que je pouvais peut-être améliorer mes techniques pour trouver d'autres offres d'emploi" (Romane, bac +4 , histoire). L'accompagnement, aménagé en différentes étapes avec des objectifs associés, leur est apparu a priori efficace et pouvant leur apporter des solutions, d'autant plus qu'il était adapté aux jeunes diplômés : "C'était pour des jeunes diplômés. Je pensais qu'il allait y avoir plus de suivi et de connaissances par rapport aux métiers..." (Florian, bac +3 , design). L'accompagnement répond donc à une attente forte des jeunes. La plupart déclarent par ailleurs qu'ils l'auraient suivi de la même manière sans l'existence de l'allocation : "Je n'aurais pas eu l'allocation, je me serais quand même inscrite les yeux fermés, parce que j'avais vraiment besoin de savoir comment m'y prendre pour rechercher et pour trouver. C'est ce qui m'importait le plus» (Jade, bac + 5, environnement).

Les consultants décrivent également des bénéficiaires impliqués. Des jeunes moins motivés ou assidus sont évoqués comme des cas minoritaires ; les abandons ou résiliations de contrat sont présentés comme marginaux ${ }^{15}$. La manière dont les jeunes plébiscitent l'accompagnement individualisé, sans y voir principalement une contrepartie de l'allocation et un contrôle de leur recherche d'emploi, montre que la crainte de l'assistanat est peu fondée pour ce public.

Les accompagnements ont été de durées variables, entre un mois et une année ${ }^{16}$. À l'issue du dispositif, $54 \%$ des jeunes ont trouvé un emploi ${ }^{17} .14 \%$ ont repris des études ou une formation, face à la nécessité de compléter sa formation initiale ou dans un but de réorientation professionnelle. Enfin, un quart des jeunes a été accompagné un an sans trouver de solution. Tous les bénéficiaires n’ont donc pas réussi à s’insérer sur le marché du travail, mais quelle que soit leur situation, ils sont nombreux à considérer que le suivi réalisé à l'Apec leur a été bénéfique. En effet, à la proposition "L'accompagnement à l'Apec a été très utile à ma recherche d'emploi ", $79 \%$ des répondants affirment être "plutôt ou tout à fait d'accord ». Pour les diplômés ayant trouvé du travail durant le RCA-JD, l'accompagne-

15. $5 \%$ des jeunes ont interrompu l'accompagnement avant la fin pour des raisons personnelles ou parce qu'il ne correspondait plus à leurs attentes (soit 8 jeunes sur les 165 répondants à l'enquête par questionnaire).

16. $30 \%$ des 276 jeunes ont été accompagnés moins de 6 mois, $43 \%$ entre 7 et 11 mois et $27 \%$ une année complète (Données Apec).

17. Il s'agit d'un emploi qualifié et à temps plein pour 8 jeunes sur 10, durable pour 6 jeunes sur 10 (CDI contrat à durée indéterminée, fonctionnaire, à son compte, $\mathrm{CDD}$ - contrat à durée déterminée - > 6 mois). Les trois quarts considèrent que cet emploi leur permet de se réaliser professionnellement, $60 \%$ s'estiment normalement ou bien payés et $53 \%$ embauchés à leur niveau de compétence (enquête par questionnaire). 
ment les a principalement aidés à "prendre confiance en eux", "persévérer dans les moments difficiles", les a "formés à la recherche d'emploi ( (enquête par questionnaire).

Ainsi, pour de nombreux jeunes ayant trouvé un emploi, l'accompagnement a facilité leur insertion professionnelle. Pour certains, il a même joué un rôle décisif dans l'accès à l'emploi recherché : " [Sans l'accompagnement] je pense que j’aurais lâché l'affaire et que je serais peut-être caissière (...) Pourquoi? Parce que javais un suivi et une motivation, alors quà Pôle emploi ils estimaient quiavec un bac +3 , on pouvait totalement sen sortir et être autonome " (Léa, bac +3 , agroalimentaire), "J'aurais pris n’importe quoi tellement j'avais envie de trouver du travail (...) Ça mia principalement redonné confiance en moi (...) Ça m'a permis aussi de retravailler correctement les bases $d u C V$, de la lettre de motivation et de mon projet professionnel» (Chloé, bac + 5, droit). Quant aux jeunes toujours en recherche d'emploi, ils soulignent également l'intérêt de ce dispositif. Ils expliquent en avoir retiré des acquis profitables pour la suite. Ils ont également apprécié d'être soutenus durant cette période souvent difficile à vivre : "Quand on recherche un premier emploi pendant plus d'un an et demi, on est assez esseulé, c'est assez dur psychologiquement. Je trouve que de toute façon, c'était vraiment bienvenu et que ça permettait d'avoir un contact social, vraiment quelqu'un qui était là pour vous remonter le moral, pour vous assister " (Noémie, bac + 3, design).

\section{Conclusion}

Le RCA-JD (Revenu Contractualisé d'Autonomie pour les Jeunes Diplômés) a donc touché un public particulier de jeunes possédant une licence, un master ou un diplôme d'écoles de commerce, d'ingénieur ou de spécialité... dans des domaines très variés. Les trois quarts avaient déjà 23 ans, étaient souvent issus de milieux plutôt favorisés et rencontraient de réelles difficultés dans la recherche du premier emploi.

Ces jeunes ne font donc pas partie des $65 \%$ des sortants de l'enseignement supérieur ayant connu des "trajectoires d'accès rapide et durable à l'emploi » mais de ceux connaissant des "trajectoires d'accès différé", de "retour en formation", de "décrochage " ou "d'éloignement de l'emploi" (Céreq, 2009). Pour ces jeunes, l'aide financière de l'Etat est venue remplacer ou compléter les ressources issues des transferts familiaux et, souvent, de revenus d'appoint du travail. La somme mensuelle de 250 euros, la plupart du temps bienvenue, a été fondue dans le budget global, participant tout autant aux frais de recherche d'emploi qu'aux dépenses de la vie quotidienne, et à un début d'indépendance financière très appréciée par ces jeunes adultes.

Mais elle est apparue inégalement adaptée à la diversité des situations individuelles. Versée sans conditions de ressources, elle a parfois profité à des jeunes de milieux favorisés vivant chez leurs parents. Son montant, plus proche de celui d'une bourse étudiante que de celui des minima sociaux, a été jugé correct par la plupart de ceux vivant chez leurs parents, mais trop faible dans l'absolu pour favoriser l'indépendance matérielle, facteur central d'une réelle autonomie de la jeunesse. 
Paradoxalement, l'accompagnement à la recherche d'emploi, qui n'aurait dû être qu'une contrepartie de l'allocation, répond à des attentes fortes.

Dès 2006, le rapport Hetzel (2006) pointe la faiblesse de l'action publique en matière d'insertion des diplômés du supérieur. Cela conduit à introduire l'insertion comme nouvelle mission des universités dans la loi LRU ${ }^{18}$, et à créer les Bureaux d'Aide à l'Insertion Professionnelle (BAIP). L'importance accordée à l'accompagnement, par les bénéficiaires du RCA, montre à la fois l'insuffisante montée en charge de ces bureaux et les carences du service public de l'emploi vis-à-vis des diplômés du supérieur, en $2011^{19}$.

Pour autant, cette expérimentation offre une réponse incomplète aux besoins d'aide à l'insertion professionnelle des diplômés de l'enseignement supérieur. Déployé dans un espace vacant de la protection sociale, pour des publics ni en emploi ni en formation, ni indemnisés, ni éligibles aux bourses étudiantes ou aux minima sociaux, ce dispositif exclut des jeunes indemnisés, plus âgés, non inscrits à Pôle emploi... pour lesquels l'accompagnement aurait du sens. Il contribue seulement à segmenter les interventions publiques en direction de la jeunesse, pour ne pas bouleverser un système de soutien aux ressources des jeunes, structuré selon le statut. Le RCA-JD constitue un petit pas de côté et non en avant dans la réforme du système de soutien aux ressources des jeunes, bien loin du "système cible" du Livre vert " de soutien à l'autonomie des jeunes ne distinguant pas, dès le départ, les droits des jeunes en formation, en emploi ou sans activité".

Au final, l'évaluation du RCA-JD éclaire surtout les difficultés rencontrées par des jeunes pourtant très diplômés à trouver un premier emploi, et le besoin d'accompagnement de cette catégorie de la jeunesse rarement prioritaire pour l'action publique.

\section{Bibliographie}

AFIJ (2012), Identification des profils de jeunes issus de l'enseignement supérieur rencontrant des difficultés d'insertion professionnelle.

Barbier J.-C. (2006), Analyse comparative de l'activation de la protection sociale en France, Grande-Bretagne, Allemane et Danemark, dans le cadre des lignes directrices de la Stratégie Européenne pour l'Emploi, rapport de recherche pour la Dares, Centre d'Etudes de l'Emploi.

18. Loi no 2007-1199 du 10 août 2007 relative aux libertés et responsabilités des universités.

19. Depuis cette date, des changements sont intervenus. Suite à l'Accord national interprofessionnel du 12 juillet 2011, l'Etat et les partenaires sociaux ont redéfini les missions de service public de l'Apec. Elles englobent désormais la sécurisation des parcours professionnels des jeunes diplômés issus de l'enseignement supérieur, à côté de celle des cadres. De même, les BAIP ont poursuivi leur développement. 
Bocquet A. (2000), Proposition de loi tendant à créer une allocation d'autonomie pour les jeunes de 16 à 25 ans, Assemblée nationale, $n^{\circ} 2737,21 / 11 / 2000$ et Sénat $n^{\circ} 134$, annexe au procès-verbal de la séance du 12/12/2000. Loi $n^{\circ} 2001-582$ du 4 juillet 2001 relative à la mise en place d'une allocation d'autonomie pour les jeunes de 16 à 25 ans.

Brin H. (2001), Familles et insertion économique et sociale des adultes de 18 à 25 ans, Conseil économique et social, Synthèse de l'avis présenté au nom de la section des Affaires sociales, 27-28 mars.

Centre d'Analyse Stratégique (2007), Les dotations en capital pour les jeunes, Boissard S., Rapport au Premier ministre, La Documentation française, Paris.

Céreq (2012), Quand l'école est finie, Génération 2007, enquête 2010, Marseille.

Céreq (2009), De l'enseignement supérieur à l'emploi : voies rapides et chemins de traverses, $\mathrm{Nef} \mathrm{n}^{\circ} 43$, octobre, Marseille.

Chevalier T. (2012), L'état providence et les jeunes, L'Harmattan, Paris, 188 p.

Cicchelli V. (2012), « Politiques de soutien à l'autonomie. Quinze ans de préconisations inabouties ", pp. 65-81, in Mucchielli L. (Dir.), Politiques de jeunesse : le grand malentendu, Champ social Editions, Nîmes, 242 p.

Commissariat général au Plan (2001), Jeunesse, le devoir d'avenir, Rapport de la commission présidée par Dominique Charvet, Paris, La Documentation française.

Commissariat général au Plan (2002), Pour une autonomie responsable et solidaire, Rapport au Premier ministre, De Foucauld J.-B., Roth N., Commission nationale pour l'autonomie des jeunes, avril.

Dmitrijeva et alii (2013), Comment améliorer la qualité des emplois salariés exercés par les étudiants ?, 2013/5, Rapport de recherche, Tepp.

Fondation Terra Nova (2010), L'autonomie des jeunes au service de l'égalité, novembre, $48 \mathrm{p}$.

Galland O. (2011) Sociologie de la jeunesse, Armand Colin « U », Paris, 256 p.

Haut-Commissariat à la Jeunesse (HCJ), (2009), Livre Vert, Commission sur la politique de la jeunesse, France, Juillet, $213 \mathrm{p}$.

Hetzel P. (2006), De l'université à l'emploi, Rapport Final, Commission du débat national "Université-emploi », 108 p.

Moncel N. (2012), "Quelle qualité d'emploi pour les jeunes diplômés du supérieur ? ", Revue Formation Emploi, n 117, Janvier-Mars, pp. 69-87.

Nicole-Drancourt C., Roulleau-Berger L. (2006), Linsertion des jeunes en France, PUF, Paris, $126 \mathrm{p}$. 
Palier B., Surel Y. (2010), L'explication du changement dans l'analyse des politiques publiques, L'Harmattan, coll. "Logiques politiques ».UNEF (2009), Mettre les jeunes en sécurité sociale. Pour une allocation d'autonomie, Volet financier du projet de statut des jeunes en formation et en insertion, mars.

Van de Velde C. (2012), «Soutenir l'autonomie des jeunes majeurs : puissance ou impuissance du politique », pp. 53-64, in Mucchielli L. (Dir.), Politiques de jeunesse : le grand malentendu, Champ Social, Nîmes, 242 p.

Van De Velde C. (2008), Devenir adulte. Sociologie comparée de la jeunesse en Europe, PUF, «Le Lien social », Paris, 278 p.

Vulbeau A. (2012), "Contrepoint, insertion des jeunes et accompagnement ", in L'accompagnement social vers l'emploi, Informations sociales, $\mathrm{n}^{\circ} 169$, Ed. Cnaf. 